\title{
MAGNETIC PROPERTIES IN Mn PURE POWDER
}

\author{
A. Szlaferek, J. Baszyński, S. Waplak \\ Institute of Molecular Physics, Polish Academy of Sciences \\ Smoluchowskiego 17, 60-179 Poznań, Poland \\ AND K. JóżWIAK \\ University of Technology, Pl. Curie 5, 60-965 Poznań, Poland
}

\begin{abstract}
The structural and magnetic properties of deformed powder of Mn obtained by mechanical grinding have been investigated using X-ray diffraction and magnetic measurements. The small values of ferromagnetic moment of Mn after grinding are attributed to the formation of a deformed phase at the surface of grains with enlargement of the interatomic spacings at the grains boundaries.
\end{abstract}

PACS numbers: 75.50.-y, 75.50.Tt

\section{Introduction}

Mechanical grinding is a process commonly used to produce ceramic magnetic materials and enables one to make powders of different dispersions relatively easily. However, it is known that mechanical grinding is a process, during which a single phase is subjected to a transformation from crystalline to amorphous-like structure.

Manganese metals are complicated systems [1]: below $1025 \mathrm{~K}, \alpha$-Mn crystallizes in the $\mathrm{A} 12$ structure, possessing a complex cubic unit cell with 58 atoms. $\alpha-\mathrm{Mn}$ is antiferromagnet with the Néel temperature of $95 \mathrm{~K}$ [1]. The spin structures"are quite complicated. There exists a realistic model with four nonequivalent types of sites in the unit cell [2], where the atomic spin polarization differs in magnitude [3, 4].

The periodicity length of the lattice is $a=8.912 \AA$. This means that for the two $\mathrm{Mn}_{\mathrm{I}}$ and eight $\mathrm{Mn}_{\text {II }}$ atoms there are 16 neighbours, for the $24 \mathrm{Mn}_{\text {III }}$ atoms 26 neighbours, and for the remaining $24 \mathrm{Mn}_{\mathrm{IV}}$ atoms 32 neighbours exist.

According to Ref. [4], every $\mathrm{Mn}_{\mathrm{I}}$ atom with positive polarization is surrounded by a tetrahedron of four $\mathrm{Mn}_{\mathrm{II}}$ atoms with negative polarization, the distance from the central atom being $2.82 \AA$. Again, every $\mathrm{Mn}_{\mathrm{II}}$ atom is surrounded by six $M_{1 I I}$ atoms at a distance of $2.48 \AA$ or $2.96 \AA$, with alternating polarization. The 24 remaining $\mathrm{Mn}_{\mathrm{IV}}$ atoms have almost negligible moments. 
The experimentally determined magnitudes of magnetic moments $m_{\mathrm{I}}, m_{\mathrm{II}}$, $m_{\mathrm{III}}$, and $m_{\mathrm{IV}}$ measured by several authors are widely different. These parameters measured by Oberteuffer et al. [3] are $1.79 \mu_{\mathrm{B}}, 1.41 \mu_{\mathrm{B}}, 1.17 \mu_{\mathrm{B}}$ and $0.09 \mu_{\mathrm{B}}$ respectively. Kasper and Roberts [4] offer two sorts of moments: first $1.54 \mu_{\mathrm{B}}, 1.54 \mu_{\mathrm{B}}$, $3.08 \mu_{\mathrm{B}}$ and $0 \mu_{\mathrm{B}}$, and second $2.5 \mu_{\mathrm{B}}, 2.5 \mu_{\mathrm{B}}, 1.7 \mu_{\mathrm{B}}$ and $0 \mu_{\mathrm{B}}$. Other values are given by Kunitomi et al. [5] namely: $1.35 \mu_{\mathrm{B}}, 1.35 \mu_{\mathrm{B}}, 0.99 \mu_{\mathrm{B}}$ and $0.22 \mu_{\mathrm{B}}$, respectively.

The details of generation and motion of dislocations under mechanical attrition, as well as their annihilation by recovery and recrystallization, seem to play a crucial role in the formation and properties of ball-milled nanocrystalline materials.

In this study deformed Mn powders were prepared from commercial elemental $\mathrm{Mn}$ in a ball mill. The powders were studied by X-ray diffraction and magnetic measurements.

\section{Experimental results}

For the experiments, we used commercial Mn plates*. Before milling, the Mn lumps were first crushed in a agate mortar to produce powder with a grain size smaller than $0.2 \mathrm{~mm}$. The ball milling was performed in a standard laboratory mill (Janetzki-KM1) using agate balls. A chemical analysis of the powder was done by the electron probe microanalysis method using a JEOL-JXA 50A. The analysis revealed mainly $\mathrm{Mn}$; less than 0.01 at \% additional $\mathrm{Si}$ was found.

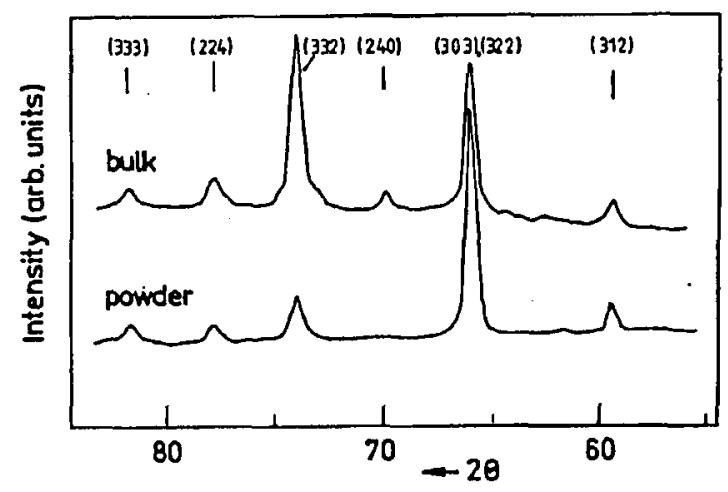

Fig. 1. X-ray diffraction patterns from $\mathrm{Mn}$ in bulk and powder form.

The X-ray diffraction patterns were taken with IIZG-4 diffractometer using Cr $K_{\alpha}$ radiation. The spectrum of the powder sample reveals only the Bragg reflections of $\alpha$-Mn (Fig. 1). The intensity of the peaks and interplanar spacings correspond to ASTM diffraction data. The difference between the ratio of the intensity peaks $(332) /(303)$ in spectra taken from bulk and powder samples results from a special texture of the bulk Mn prepared by electroplating process [6] apparently absent in the powder.

*Specpure Johnson-Matthey Chemicals Ltd. 

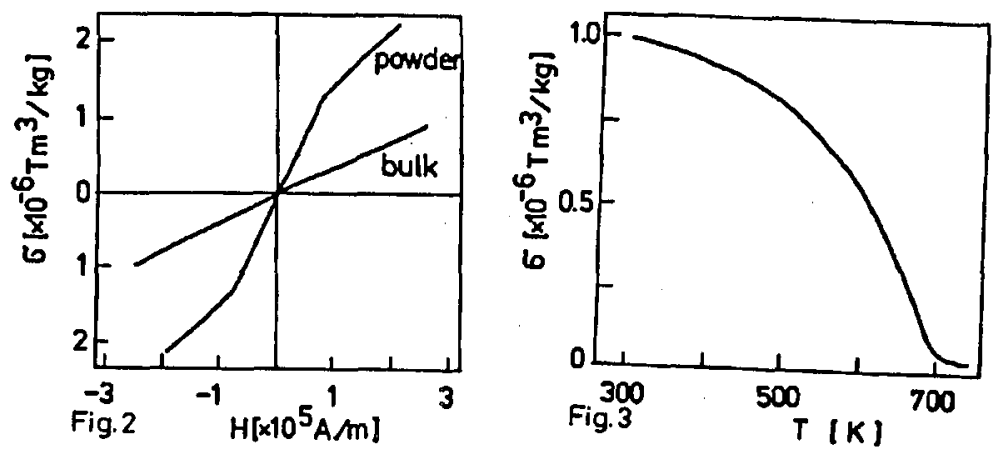

Fig. 2. Hysteresis loop for $\mathrm{Mn}$ in bulk and powder form.

Fig. 3. Magnetization vs. temperature for Mn powder.

For the magnetic measurements the powder was compacted by isostatic pressing in the form of cylindres, $4 \mathrm{~mm}$ in a diameter and $6 \mathrm{~mm}$ in a height. The magnetic hysteresis loops at room temperature (sce Fig. 2) were measured with a VSM. The magnetization processes in bulk and powder form differ remarkably. Figure 3 shows the magnetization versus temperature curves for a $\mathrm{Mn}$ powder sample carried out in the temperature range from $300 \mathrm{~K}$ to $800 \mathrm{~K}$ using a Faraday balance. The room temperature magnetization in this sample was $1.0 \times 10^{-6} \mathrm{~T} \mathrm{~m}^{3} / \mathrm{kg}$ and Curie temperature was about $690 \mathrm{~K}$. ESR spectra, were recorded by X-band spectrometer, taken by us for bulk and powder Mn samples with a grain size order of $45 \mu \mathrm{m}$. The ESR signal with the linewidth $48 \mathrm{mT}$, $g=1.99 \pm 0.01$ and Lorentzian line-shape was observed only for grained samples.

\section{Discussion}

Two mechanisms permit a satisfactory interpretation of above unusual experimental results relating to magnetic properties of grained Mn samples:

1. The fact that the point for Mn lies on the steep part of Slater-Bethe's curve agrees nicely with the fact that the appearance of ferromagnetism is accompanied by an enlargement of the interatomic distances at grains boundaries. Hence, we attribute the ferromagnetic phase to the deformed surface of the Mn grains.

2. The above magnetic behaviour of $\mathrm{Mn}$ powder has been attributed to a ferrimagnetic structure similar to that existing in the ferrites. The basic structure is antiferromagnetic with two ferromagnetic sublattices, aligned with net magnetic moments antiparallel, but with a different magnetization for each sublattice (different density of atoms), thereby giving a net magnetization detectable by magnetic measurements. The concept of ferrimagnetism has been generalized over the original ferrimagnetic behaviour of the antiferromagnetics. Néel [7] favours the view that defects or vacancies assume an ordered state below a certain critical temperature, resulting in an unequal number of atoms for each sublattice, thereby producing ferrimagnetism. In the grained $\mathrm{Mn}$ samples studied here crystalline defects are present leading to a sublattice unequality. 


\section{References}

[1] R.S. Tebble, D.J. Craik, Magnetic Materials, Interscience, London 1969.

[2] W.G. Wyckoff, Crystal Slructures, Vol. 1, Interscience, New York 1965.

[3] J.A. Oberteufter, J.A. Marcus, L.II. Schwartz, G.P. Felcher, Phys. Lelt. A 28, 267 (1968).

[4] J.S. Kasper, B.W. Roberts, Phys. Rev. 101, 537 (1956).

[5] N. Kunitomi, Y. Yamada, Y. Nakai, Y. Fujii, J. Appl. Phys. 40, 1265 (1969).

[6] H.P. Klug, L.E. Alexander, X-ray Diffraction Procedures, Wiley-Interscience, New York 1965.

[7] L. Néel, Rev. Mod. Phys. 25, 356 (1955). 